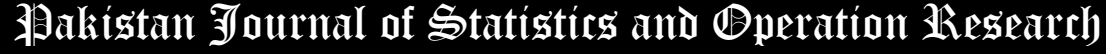

\section{A Generalization of Reciprocal Exponential Model: Clayton Copula, Statistical Properties and Modeling Skewed and Symmetric Real Data Sets}

\author{
M. M. Mansour ${ }^{1,2}$, Nadeem Shafique Butt ${ }^{3 *}$, Haitham M. Yousof ${ }^{2 *}$, \\ S. I. Ansari ${ }^{4}$ and Mohamed Ibrahim ${ }^{5}$ \\ ${ }^{1}$ Department of MIS, Yanbu, Taibah University, Saudi Arabia. \\ ${ }^{2}$ Department of Statistics, Mathematics and Insurance, Benha University, Egypt. \\ MAHMOUD.MANSOUR@fcom.bu.edu.eg , haitham.yousof@fcom.bu.edu.eg \\ ${ }^{3}$ Department of Family and Community Medicine, King Abdul Aziz University, Jeddah, Kingdom of Saudi \\ Arabia.nshafique@kau.edu.sa \\ ${ }^{4}$ Department of Business Administration, Azad institute of Engineering and Technology, Lucknow, \\ India.saiful.islam.ansari@gmail.com \\ ${ }^{5}$ Department of Applied Statistics and Insurance, Faculty of Commerce, Damietta University, Damietta, Egypt. \\ mohamed_ibrahim@du.edu.eg \\ * Corresponding Author.
}

\begin{abstract}
We introduce a new extension of the reciprocal Exponential distribution for modeling the extreme values. We used the Morgenstern family and the clayton copula for deriving many bivariate and multivariate extensions of the new model. Some of its properties are derived. We assessed the performance of the maximum likelihood estimators (MLEs) via a graphical simulation study. The assessment was based on the sample size. The new reciprocal model is employed for modeling the skewed and the symmetric real data sets. The new reciprocal model is better than some other important competitive models in statistical modeling.
\end{abstract}

Keywords: Reciprocal Exponential Distribution; Morgenstern Family Moments; Clayton Copula; Estimation; Simulations; Odd Log-Logistic Family.

\section{Introduction}

A random variable (RV) $Y$ is said to have the reciprocal Exponential (RE) distribution if its probability density function (P-D-F) and cumulative distribution function (C-D-F) are given by

$$
g_{c_{3}}(y)=\left.c_{3} y^{-2} \mathrm{e}^{-\frac{c_{3}}{y}}\right|_{y \geq 0}
$$

and

$$
G_{c_{3}}(y)=\left.\mathrm{e}^{-\frac{c_{3}}{y}}\right|_{y \geq 0},
$$

where $c_{3}>0$ is a scale parameter. The P-D-F in (1) is a special case from the well-known reciprocal Weibull (RW) model. The RW model is also known as the Fréchet distribution (see Fréchet (1927) ) and also contains the reciprocal Rayleigh (RR) model as a special case. Cordeiro (2017) proposed a new class of continuous distributions called the generalized odd log-logistic-G (GOLL-G) family with two extra shape parameters $c_{1}>0$ and $c_{2}>0$. For an arbitrary baseline C-D-F $G_{\xi}(y)$, the C-D-F of the the GOLL-G family is given by

$$
F_{c_{1}, c_{2}, \xi}(y)=\frac{G_{\xi}(y)^{c_{1} c_{2}}}{G_{\xi}(y)^{c_{1} c_{2}}+\left[1-G_{\xi}(y)^{c_{2}}\right]^{c_{1}}} .
$$

The P-D-F corresponding to (3) is given by 




For $c_{2}=1$ we get the OLL-G family Gleaton and Lynch (2006). For $c_{1}=1$ we get the Proportional reversed hazard rate $\mathrm{G}$ family Gupta and Gupta (2007). Here, we define a new RE model based on Cordeiro (2017)] called generalized odd log-logistic RE (GOLL-RE) family and provide some plots of its P-D-F and hazard rate function (H-R-F) $\left[h_{c_{1}, c_{2}, c_{3}}(y)\right]$. The GOLL-RE C-D-F is given by

$$
F_{c_{1}, c_{2}, c_{3}}(y)=\frac{\mathrm{e}^{-c_{1} c_{2} \frac{c_{3}}{y}}}{\mathrm{e}^{-c_{1} c_{2} \frac{c_{3}}{y}}+\left(1-\mathrm{e}^{-c_{2} \frac{c_{3}}{y}}\right)^{c_{1}}} .
$$

The P-D-F corresponding to (5) is given by

$$
f_{c_{1}, c_{2}, c_{3}}(y)=\frac{c_{1} c_{2} c_{3} y^{-2} \mathrm{e}^{-c_{1} c_{2} \frac{c_{3}}{y}}\left(1-\mathrm{e}^{-c_{2} \frac{c_{3}}{y}}\right)^{c_{1}-1}}{\left[\mathrm{e}^{-c_{1} c_{2} \frac{c_{3}}{y}}+\left(1-\mathrm{e}^{-c_{2} \frac{c_{3}}{y}}\right)^{c_{1}}\right]^{2}} .
$$

The H-R-F for the new model can be get from $f_{c_{1}, c_{2}, c_{3}}(y) /\left[1-F_{c_{1}, c_{2}, c_{3}}(y)\right]$. Let $c=\inf \left\{\left.y\right|_{G(y ; \xi)>0}\right\}$, the asymptotics of the C-D-F, P-D-F and H-R-F as $y \rightarrow c$ are given by

and

$$
\begin{gathered}
\left.F_{c_{1}, c_{2}, c_{3}}(y) \sim \mathrm{e}^{-c_{1} c_{2} \frac{c_{3}}{y}}\right|_{y \rightarrow c}, \\
\left.f_{c_{1}, c_{2}, c_{3}}(y) \sim c_{1} c_{2} y^{-2} \mathrm{e}^{-c_{1} c_{2} \frac{c_{3}}{y}}\right|_{y \rightarrow c}
\end{gathered}
$$

The asymptotics of C-D-F, P-D-F and H-R-F as $y \rightarrow \infty$ are given by

$$
\left.h_{c_{1}, c_{2}, c_{3}}(y) \sim c_{1} c_{2} y^{-2} \mathrm{e}^{-c_{1} c_{2} \frac{c_{3}}{y}}\right|_{y \rightarrow c}
$$

$$
\begin{gathered}
1-\left.F_{c_{1}, c_{2}, c_{3}}(y) \sim\left(c_{2}\left\{1-\mathrm{e}^{-\frac{c_{3}}{y}}\right\}\right)^{c_{1}}\right|_{y \rightarrow \infty}, \\
\left.f_{c_{1}, c_{2}, c_{3}}(y) \sim c_{1} c_{2} y^{-2} \mathrm{e}^{-\frac{c_{3}}{y}}\left(1-\mathrm{e}^{-\frac{c_{3}}{y}}\right)^{c_{1}-1}\right|_{y \rightarrow \infty}
\end{gathered}
$$

and

$$
\left.h_{c_{1}, c_{2}, c_{3}}(y) \sim \frac{c_{1} y^{-2} \mathrm{e}^{-\frac{c_{3}}{y}}}{1-\mathrm{e}^{-\frac{c_{3}}{y}}}\right|_{y \rightarrow \infty}
$$

Some plots of the GOLL-RE P-D-F and H-R-F are given in Figure 1 (see Appendix A for all Tables and Appendix B for all Figures) to illustrate some of its characteristics. For simulation of this new model, we obtain the quantile function (QF) of $y$ (by inverting (5)), say $y_{u}=Q(u)=F^{-1}(u)$, as

$$
y_{u}=c_{3}\left(-\ln \left\{\left[\frac{\left(\frac{u}{1-u}\right)^{\frac{1}{c_{1}}}}{1+\left(\frac{u}{1-u}\right)^{\frac{1}{c_{1}}}}\right]^{\frac{1}{c_{2}}}\right\}\right)^{-1} .
$$

Equation (7) is used for simulating the new model (see Section 5). Some important extensions of the RE model have been developed and studied Yousof et al., (2015), Yousof et al., (2016), Korkmaz et al., (2017), Brito et al., (2017), Hamedani et al., (2017), Cordeiro et al., (2018), Yousof et al., (2018 a), Chakraborty et al., (2018), Hamedani et al., (2018), Korkmaz et al., (2018), Yousof et al., (2018 b), Hamedani et al., (2019), Goual and Yousof (2019) and Korkmaz et al., (2019), among others.

\section{Copula}

\subsection{Via Morgenstern family}

Consider the C-D-F of the Morgenstern $\left(\left.\lambda\right|_{(|\lambda| \leq 1)}\right)$ family which has the following form

$$
\left.F_{\lambda}\left(y_{1}, y_{2}\right)\right|_{(|\lambda| \leq 1)}=F_{1}\left(y_{1}\right) F_{2}\left(y_{2}\right)\left\{1+\lambda\left[1-F_{1}\left(y_{1}\right)\right]\left[1-F_{2}\left(y_{2}\right)\right]\right\}
$$

setting

$$
F_{c_{1}, c_{2}, c_{3}}\left(y_{1}\right)=\frac{\mathrm{e}^{-c_{1} c_{2} \frac{c_{3}}{y_{1}}}}{\mathrm{e}^{-c_{1} c_{2} \frac{c_{3}}{y_{1}}}+\left(1-\mathrm{e}^{-c_{2} \frac{c_{3}}{y_{1}}}\right)^{c_{1}}}
$$


and

$$
F_{a_{1}, a_{2}, a_{3}}\left(y_{2}\right)=\frac{\mathrm{e}^{-a_{1} a_{2} \frac{a_{3}}{y_{1}}}}{\mathrm{e}^{-a_{1} a_{2} \frac{a_{3}}{y_{2}}+\left(1-\mathrm{e}^{-a_{2} \frac{a_{3}}{y_{2}}}\right)^{a_{1}}},}
$$

then we have a 7-dimensional bivariate GOLL-RE model model as

Where

$$
\begin{aligned}
& F_{\underline{\Xi}}\left(y_{1}, y_{2}\right)=\frac{\mathrm{e}^{-c_{1} c_{2} \frac{c_{3}}{y_{1}}}}{\mathrm{e}^{-c_{1} c_{2} \frac{c_{3}}{y_{1}}}+\left(1-\mathrm{e}^{-c_{2} \frac{c_{3}}{y_{1}}}\right)^{c_{1}}} \frac{\mathrm{e}^{-a_{1} a_{2} \frac{a_{3}}{y_{1}}}}{\mathrm{e}^{-a_{1} a_{2} \frac{a_{3}}{y_{2}}}+\left(1-\mathrm{e}^{-a_{2} \frac{a_{3}}{y_{2}}}\right)^{a_{1}}} \\
& \times\left(1+\lambda\left\{\left[1-\frac{\mathrm{e}^{-c_{1} c_{2} \frac{c_{3}}{y_{1}}}}{\mathrm{e}^{-c_{1} c_{2} \frac{c_{3}}{y_{1}}}+\left(1-\mathrm{e}^{-c_{2} \frac{c_{3}}{y_{1}}}\right)^{c_{1}}}\right]\left[1-\frac{\mathrm{e}^{-a_{1} a_{2} \frac{a_{3}}{y_{1}}}}{\mathrm{e}^{-a_{1} a_{2} \frac{a_{3}}{y_{2}}}+\left(1-\mathrm{e}^{-a_{2} \frac{a_{3}}{y_{2}}}\right)^{a_{1}}}\right]\right\}\right),
\end{aligned}
$$

$$
\underline{\underline{\Xi}}=|\lambda| \leq 1, c_{1}>0, c_{2}>0, c_{3}>0, a_{1}>0, a_{2}>0, a_{3}>0 .
$$

\subsection{Via clayton copula}

\subsubsection{The bivariate extension}

The bivariate extension via clayton copula can be considered as a weighted version of the clayton copula, which is of the form

$$
C(u, v)=\left[u^{-\left(\delta_{1}+\delta_{2}\right)}+v^{-\left(\delta_{1}+\delta_{2}\right)}-1\right]^{-\frac{1}{\delta_{1}+\delta_{2}}} .
$$

This is indeed a valid copula. Next, let us assume that $Y \sim \operatorname{GOLL}-\operatorname{RE}\left(c_{1}, c_{2}, c_{3}\right)$ and $Z \sim \operatorname{GOLL}-\operatorname{RE}\left(a_{1}, a_{2}, a_{3}\right)$.Then, setting

and

$$
u=\frac{\mathrm{e}^{-c_{1} c_{2} \frac{c_{3}}{y}}}{\mathrm{e}^{-c_{1} c_{2} \frac{c_{3}}{y}}+\left(1-\mathrm{e}^{-c_{2} \frac{c_{3}}{y}}\right)^{c_{1}}}
$$

$$
v=\frac{\mathrm{e}^{-a_{1} a_{2} \frac{a_{3}}{z}}}{\mathrm{e}^{-a_{1} a_{2} \frac{a_{3}}{z}}+\left(1-\mathrm{e}^{-a_{2} \frac{a_{3}}{z}}\right)^{a_{1}},}
$$

the associated bivariate C-D-F of the GOLL-RE type distribution will be

Where

$$
H(y, z)=\left\{\left[\frac{\mathrm{e}^{-c_{1} c_{2} \frac{c_{3}}{y}}}{\mathrm{e}^{-c_{1} c_{2} \frac{c_{3}}{y}}+\left(1-\mathrm{e}^{-c_{2} \frac{c_{3}}{y}}\right)^{c_{1}}}\right]^{-\left(\delta_{1}+\delta_{2}\right)}+\left[\frac{\mathrm{e}^{-a_{1} a_{2} \frac{a_{3}}{z}}}{\mathrm{e}^{-a_{1} a_{2} \frac{a_{3}}{z}}+\left(1-\mathrm{e}^{-a_{2} \frac{a_{3}}{z}}\right)^{a_{1}}}\right]^{-\left(\delta_{1}+\delta_{2}\right)}-1\right\}^{-\frac{1}{\delta_{1}+\delta_{2}}} .
$$

$$
\left(\delta_{1}+\delta_{2}\right) \geq 0,, c_{1}>0, c_{2}>0, c_{3}>0, a_{1}>0, a_{2}>0, a_{3}>0 .
$$

\subsubsection{The Multivariate extension}

A straightforward $d$-dimensional extension IRom the above will be

$$
\left.H\left(y_{1}, y_{2}, \cdots, y_{d}\right)=\left\{\sum_{i=1}^{d}\left[\frac{\mathrm{e}^{-c_{1 i} c_{2 i} c_{3 i}}}{\mathrm{e}^{-c_{1 i} c_{2 i} c_{3 i}} \frac{y_{i}}{y_{i}}+\left(1-\mathrm{e}^{-c_{2 i} \frac{c_{3 i}}{y_{i}}}\right)}\right]^{c_{1 i}}\right]^{-\left(\delta_{1}+\delta_{2}\right)}+1-d\right\}^{-1 /\left(\delta_{1}+\delta_{2}\right)},
$$

where where $\left(\delta_{1}+\delta_{2}\right) \geq 0, c_{1 i}>0, c_{2 i}>0, c_{3 i}>0$. Future works may be allocated for studying the new bivariate and multivariate extensions of the GOLL-RE model.

\section{Properties}

\subsection{Representations}

Based on Cordeiro et al. (2016), the P-D-F in (6) can be expressed as

$$
f(y)=\sum_{k_{1}=0}^{\infty} \mathbf{C}_{k_{1}} \pi_{\left(1+k_{1}\right)}\left(y ; c_{3}\right),
$$


where

$$
\begin{gathered}
\mathbf{C}_{k_{1}}=\frac{c_{1} c_{2}}{1+k_{1}} \sum_{k_{2}, k_{3}=0}^{\infty} \sum_{k_{1}=0}^{k_{4}}(-1)^{k_{3}+k_{1}+k_{4}}\left(\begin{array}{l}
-2 \\
k_{2}
\end{array}\right) \\
\times\left(\begin{array}{l}
-c_{1}\left(k_{2}+1\right) \\
k_{3}
\end{array}\right)\left(\begin{array}{l}
c_{1} c_{2}\left(k_{2}+1\right)+c_{2} k_{3}-1 \\
k_{4}
\end{array}\right)\left(\begin{array}{l}
k_{4} \\
k_{1}
\end{array}\right),
\end{gathered}
$$

and $\pi_{\left(1+k_{1}\right)}\left(y ; c_{3}\right)$ is the P-D-F of the Fr model with scale parameter $c_{3}\left(1+k_{1}\right)$ and shape parameter $b$. So, the new P-DF in (6) can be expressed as a double linear mixture of the RE P-D-F. Then, several structural properties of the new model can be obtained from Equation (8) and those properties of the RE model. By integrating Equation (8), the C-D-F of $y$ becomes

$$
F(y)=\sum_{k_{1}=0}^{\infty} \mathbf{C}_{k_{1}} \Pi_{\left(1+k_{1}\right)}\left(y ; c_{3}\right),
$$

where $\Pi_{\left(1+k_{1}\right)}\left(y ; c_{3}\right)$ is the C-D-F of the R-E distribution with scale parameter $c_{3} k_{1}$.

\subsection{Moments and incomplete moments}

The $\mathbf{s}^{\text {th }}$ ordinary moment of $y$ is given by

then we obtain

$$
\mu_{\mathbf{s}}^{\prime}=E\left(Y^{\mathbf{s}}\right)=\int_{-\infty}^{\infty} y^{\mathbf{s}} f(y) d y,
$$

where

$$
\mu_{\mathbf{s}}^{\prime}=\sum_{k_{1}=0}^{\infty} \mathbf{C}_{k_{1}} c_{3}\left(1+k_{1}\right)^{s} \boldsymbol{\Gamma}(1-\mathbf{s}), \forall 1>\mathbf{s},
$$

$$
\left.\boldsymbol{\Gamma}\left(1+\boldsymbol{\Psi}_{1}\right)\right|_{\left(\boldsymbol{\Psi}_{1} \in \mathbb{R}^{+}\right)}=\boldsymbol{\Psi}_{1} !=\prod_{h=0}^{\boldsymbol{\psi}_{1}-1}\left(\boldsymbol{\Psi}_{1}-h\right) .
$$

The $\mathbf{s}^{\text {th }}$ incomplete moment, say $\varphi_{\mathbf{s}}(\boldsymbol{\tau})$, of $Y$ can be expressed, from (9), as

$$
\begin{aligned}
\varphi_{\mathbf{s}}(\boldsymbol{\tau})=\int_{-\infty}^{\boldsymbol{\tau}} y^{\mathbf{s}} f(y) d y=\sum_{k_{1}=0}^{\infty} k_{1} \int_{-\infty}^{\boldsymbol{\tau}} y^{\mathbf{s}} \pi_{\left(1+k_{1}\right)}\left(y ; c_{3}\right) d y \\
=\sum_{k_{1}=0}^{\infty} k_{1} c_{3}\left(1+k_{1}\right)^{\mathbf{s}} \gamma\left(1-\mathbf{s},\left(1+k_{1}\right)\left(\frac{c_{3}}{\boldsymbol{\tau}}\right)^{b}\right), \forall 1>\mathbf{s},
\end{aligned}
$$

where $\gamma\left(\boldsymbol{\Psi}_{1}, \boldsymbol{\Psi}_{2}\right)$ is the incomplete gamma function.

$$
\begin{gathered}
\left.\gamma\left(\boldsymbol{\Psi}_{1}, \boldsymbol{\Psi}_{2}\right)\right|_{\left(\boldsymbol{\Psi}_{1} \neq 0,-1,-2, \ldots\right)}=\int_{0}^{\boldsymbol{\Psi}_{2}} \boldsymbol{\tau}^{\boldsymbol{\Psi}_{1}-1} \exp (-\boldsymbol{\tau}) d \boldsymbol{\tau} \\
=\frac{\boldsymbol{\Psi}_{2}^{\boldsymbol{\Psi}_{1}}}{\boldsymbol{\Psi}_{1}}\left\{{ }_{1} \mathbf{F}_{1}\left[\boldsymbol{\Psi}_{1} ; \boldsymbol{\Psi}_{1}+1 ;-\boldsymbol{\Psi}_{2}\right]\right\} \\
=\sum_{k_{1}=0}^{\infty} \frac{(-1)^{k_{1}}}{k_{1} !\left(\boldsymbol{\Psi}_{1}+k_{1}\right)} \boldsymbol{\Psi}_{2}^{\boldsymbol{\Psi}_{1}+k_{1}},
\end{gathered}
$$

and ${ }_{1} \mathbf{F}_{1}[\because, \cdot]$ is a confluent hypergeometric function. The first incomplete moment given by (11) with $\mathbf{s}=1$ as

$$
\varphi_{1}(\boldsymbol{\tau})=\sum_{k_{1}=0}^{\infty} w_{k_{1}} c_{3}\left(1+k_{1}\right) \boldsymbol{\Gamma}\left(1-\frac{1}{b},\left(1+k_{1}\right)\left(\frac{c_{3}}{\boldsymbol{\tau}}\right)\right) .
$$

\subsection{Moment generating function (MGF)}

The MGF $M_{Y}(\boldsymbol{\tau})=E\left(e^{\boldsymbol{\tau} Y}\right)$ of $Y$ can be derived from equation (8) as

$$
M_{Y}(\boldsymbol{\tau})=\sum_{k_{1}=0}^{\infty} \mathbf{C}_{k_{1}} M_{\left(1+k_{1}\right)}\left(\boldsymbol{\tau} ; c_{3}\right),
$$

where $M_{\left(1+k_{1}\right)}\left(\boldsymbol{\tau} ; c_{3}\right)$ is the MGF of the Fr model with scale parameter $c_{3}\left(1+k_{1}\right)$, then

$$
M_{Y}(\boldsymbol{\tau})=\sum_{k_{1}=0}^{\infty} \sum_{\mathbf{s}=0}^{\infty} \mathbf{C}_{k_{1}} \frac{\boldsymbol{\tau}^{\mathbf{s}}}{\mathbf{s} !} c_{3}\left[\left(1+k_{1}\right)\right]^{\mathbf{s}} \boldsymbol{\Gamma}(1-\mathbf{s}), \forall 1>\mathbf{s} .
$$




\subsection{Residual life and reversed residual life functions}

The $\mathbf{m}^{\text {th }}$ moment of the residual life

the $\mathbf{m}^{\text {th }}$ moment of the residual life of $y$ is given by

$$
A_{\mathbf{s}}(\boldsymbol{\tau})=E\left[\left.(Y-\boldsymbol{\tau})^{\mathbf{s}}\right|_{Y>\boldsymbol{\tau}, \mathbf{s}=1,2, \ldots]}\right.
$$

Therefore,

$$
A_{\mathbf{m}}(\boldsymbol{\tau})=\frac{\int_{\boldsymbol{\tau}}^{\infty}(Y-\boldsymbol{\tau})^{\mathbf{m}} d F(y)}{1-F(\boldsymbol{\tau})}
$$

where

$$
A_{\mathbf{m}}(\boldsymbol{\tau})=\frac{c_{3}}{1-F(\boldsymbol{\tau})} \sum_{k_{1}=0}^{\infty} \zeta_{k_{1}}\left(1+k_{1}\right)^{s} \boldsymbol{\Gamma}\left(1-\mathbf{s},\left(1+k_{1}\right)\left(\frac{c_{3}}{\boldsymbol{\tau}}\right)\right), \forall 1>\mathbf{m}
$$

and

$$
\begin{gathered}
\zeta_{k_{1}}=\mathbf{C}_{k_{1}} \sum_{\mathbf{s}=0}^{\mathbf{m}}\left(\begin{array}{l}
\mathbf{m} \\
\mathbf{s}
\end{array}\right)(-\boldsymbol{\tau}), \\
\left.\Gamma\left(\boldsymbol{\Psi}_{1}, \boldsymbol{\Psi}_{2}\right)\right|_{y>0}=\int_{\boldsymbol{\Psi}_{2}}^{\infty} \boldsymbol{\tau}^{\boldsymbol{\Psi}_{1}-1} \exp (-\boldsymbol{\tau}) d \boldsymbol{\tau},
\end{gathered}
$$

The $\mathbf{m}^{\text {th }}$ moment of the reversed residual life, say

$$
\boldsymbol{\Gamma}\left(\boldsymbol{\Psi}_{1}, \boldsymbol{\Psi}_{2}\right)+\gamma\left(\boldsymbol{\Psi}_{1}, \boldsymbol{\Psi}_{2}\right)=\boldsymbol{\Gamma}\left(\boldsymbol{\Psi}_{1}\right) .
$$

uniquely determines $F(y)$. We obtain

$$
B_{\mathbf{m}}(\boldsymbol{\tau})=E\left[\left.(\boldsymbol{\tau}-Y)^{\mathbf{m}}\right|_{Y \leq \boldsymbol{\tau}, \boldsymbol{\tau}>0} \text { and } \mathbf{m}=1,2, \ldots\right]
$$

where

$$
B_{\mathbf{m}}(\boldsymbol{\tau})=\frac{\int_{0}^{\boldsymbol{\tau}}(\boldsymbol{\tau}-y)^{\mathbf{m}} d F(y)}{F(\boldsymbol{\tau})}
$$

Then, the $\mathbf{m}^{\text {th }}$ moment of the reversed residual life of $y$ becomes

$$
B_{\mathbf{m}}(\boldsymbol{\tau})=\frac{c_{3}}{F(\boldsymbol{\tau})} \sum_{k_{1}=0}^{\infty} \eta_{k_{1}}\left(1+k_{1}\right)^{\mathbf{s}} \gamma\left(1-\mathbf{s},\left(1+k_{1}\right)\left(\frac{c_{3}}{\mathbf{\tau}}\right)\right), \forall 1>\mathbf{m},
$$

$$
\eta_{k_{1}}=\mathbf{C}_{k_{1}} \sum_{\mathbf{s}=0}^{\mathbf{m}}(-1)^{\mathbf{s}}\left(\begin{array}{l}
\mathbf{m} \\
\mathbf{s}
\end{array}\right) \mathbf{\tau}^{\mathbf{m}-\mathbf{s}}
$$

\section{The MLE}

Let $y_{1}, \ldots, y_{n}$ be a random sample from the GOLL-RE distribution with parameters $c_{1}, c_{2}, c_{3}$. Let $\Theta=\left(c_{1}, c_{2}, c_{3}\right)^{\top}$ be the $3 \times 1$ parameter vector. For determining the MLE of $\Theta$, we have the $\log$-likelihood function

$$
\begin{aligned}
\ell=\ell(\Theta)= & n \log \left(c_{1} c_{2} c_{3}\right)-2 \sum_{i=1}^{n} \log \left(y_{i}\right)-c_{1} c_{2} \sum_{i=1}^{n} \frac{c_{3}}{y_{i}} \\
+ & 2 \sum_{i=1}^{n} \log \left[\mathrm{e}^{-c_{1} c_{2} \frac{c_{3}}{y_{i}}}+\left(1-\mathrm{e}^{-c_{2} \frac{c_{3}}{y_{i}}}\right)^{c_{1}}\right] \\
& +\left(c_{1}-1\right) \sum_{i=1}^{n} \log \left(1-\mathrm{e}^{-c_{2} \frac{c_{3}}{y_{i}}}\right) .
\end{aligned}
$$

The components of the score vector

$$
\mathbf{L}_{(\Theta)}=\frac{\partial \ell}{\partial \Theta}=\left(\frac{\partial}{\partial c_{1}}, \frac{\partial}{\partial c_{2}}, \frac{\partial}{\partial c_{3}}\right)^{\top},
$$

are available if needed. Setting $\mathbf{L}_{c_{1}}=\mathbf{L}_{c_{2}}=\mathbf{L}_{c_{3}}=\mathbf{0}$ and solving them simultaneously yields the MLE $\hat{c}=$ $\left(\widehat{c_{1}}, \widehat{c_{2}}, \widehat{c_{3}}\right)^{\top}$. To solve these equations, it is usually more convenient to use nonlinear optimization methods such as the quasi-Newton algorithm to numerically maximize $\ell$. For interval estimation of the parameters, we obtain the $3 \times 3$ observed information matrix

$$
J(\Theta)=\left\{\frac{\partial^{2} \ell}{\partial \mathbf{s}} \partial p\right\}\left(\forall \mathbf{s}, p=c_{1}, c_{2}, c_{3}\right)
$$


whose elements can be computed numerically.

\section{Graphical simulations}

In this Section, we can performed the simulation experiments graphically to assess of the finite sample behavior of the MLEs.

\section{The graphical assessment was based on the following algorithm:}

1. use (7) for generating 10000 samples of size $n$ from the GOLL-RE model;

2. compute the MLEs for the 1000 samples, say

$$
\left.\left(\overline{\left(c_{1}\right)_{l}}, \overline{\left(c_{2}\right)_{l}}, \overline{\left(c_{3}\right)_{l}}\right)\right|_{(i=1,2, \ldots, 10000)}
$$

3. compute the SEs of the MLEs for the 1000 samples, say

$$
\left.\left(S_{\overline{\left(c_{1}\right)_{l}}}, S_{\left.\overline{\left(c_{2}\right)}\right)_{l}}, S_{\overline{\left(c_{3}\right)_{l}}}\right)\right|_{(i=1,2, \ldots, 10000)} \text {. }
$$

The standard errors (SEs) were computed by inverting the observed information matrix.

4. compute the biases and mean squared errors given for $\mathbf{p}=c_{1}, c_{2}, c_{3}$. We repeated these steps for $n=$ $50,100, \ldots, 500$ with $c_{1}=1, c_{2}=1, c_{3}=1$, so computing biases $\left(\operatorname{Bias}_{\mathbf{p}}(n)\right)$, mean squared errors (MSEs) $\left(\operatorname{MSE}_{\mathbf{p}}(n)\right)$ for $c_{1}, c_{2}, c_{3}$ and $n=50,100, \ldots, 500$ where

and

$$
\left.\operatorname{Bias}_{\mathbf{p}}(n)\right|_{\left(\mathbf{p}=c_{1}, c_{2}, c_{3}\right)}=\frac{1}{10000} \sum_{i=1}^{10000}\left(\widehat{\mathbf{p}}_{i}-\mathbf{p}\right),
$$

$$
\left.\operatorname{MSE}_{\mathbf{p}}(n)\right|_{\left(\mathbf{p}=c_{1}, c_{2}, c_{3}\right)}=\frac{1}{10000} \sum_{i=1}^{10000}\left(\widehat{\mathbf{p}}_{i}-\mathbf{p}\right)^{2}
$$

Figures 2-4 give the biases and MSEs for $c_{1}, c_{2}$ and $c_{3} \forall n=50,100, \ldots, 500$ for the GOLL-RE model. Figures 2-4 shows how the biases and MSEs vary with respect to $n$. The broken line in Figure 4 corresponds to the biases being 0 . From Figures 2-4, the biases for each parameter are generally negative and decrease to zero as $n \rightarrow \infty$, the MSEs for each parameter decrease to zero as $n \rightarrow \infty$.

\section{Real data modeling}

We consider the Cramér-Von Mises (C-V-M) and the Anderson-Darling (A-D) and the Kolmogorov-Smirnov (KS) statistic. We compare the fits of the GOLL-RE distribution with other models such as RW, Kumaraswamy-RW (KFr), exponentiated RW (E-RW), beta-RW (B-RW), transmuted-RW (T-RW), Marshal-Olkin-RW (MO-RW) and McDonald-RW (Mc-RW) distributions given by:

E-RW :

B-RW :

$$
f_{E F r}(z)=c_{1} b c_{3} y^{-(b+1)} \exp \left[-\left(c_{3} / y\right)^{b}\right]\left\{1-\exp \left[-\left(c_{3} / y\right)^{b}\right]\right\}^{c_{1}-1} ;
$$

K-RW :

$$
f_{B F r}(z)=b c_{3} B^{-1}\left(c_{1}, c\right) y^{-(b+1)} \exp \left[-c_{1}\left(c_{3} / y\right)^{b}\right]\left\{1-\exp \left[-\left(c_{3} / y\right)^{b}\right]\right\}^{c-1} ;
$$

T-RW :

$$
f_{K R W}(z)=c_{1} c b c_{3} y^{-(b+1)} \exp \left[-c_{1}\left(c_{3} / y\right)^{b}\right]\left\{1-\exp \left[-c_{1}\left(c_{3} / y\right)^{b}\right]\right\}^{c-1} ;
$$

MO-RW :

$$
f_{T-R W}(z)=b c_{3} y^{-(b+1)} \exp \left[-\left(c_{3} / y\right)^{b}\right]\left\{1+c_{1}-2 c_{1} \exp \left[-\left(c_{3} / y\right)^{b}\right]\right\} ;
$$

Mc-RW :

$$
f_{M O-R W}(z)=c_{1} b c_{3} y^{-(b+1)} \exp \left[-\left(c_{3} / y\right)^{b}\right]\left\{c_{1}+\left(1-c_{1}\right) \exp \left[-\left(c_{3} / y\right)^{b}\right]\right\}^{-2} ;
$$

OLLE-RW:

$$
\begin{aligned}
& \quad f_{M c-R W}(z)=\lambda b c_{3} y^{-(b+1)} B^{-1}\left(c_{1}, c\right) \exp \left[-\left(c_{3} / y\right)^{b}\right]\left(\exp \left[-\left(c_{3} / y\right)^{b}\right]\right)^{c_{1} \lambda-1} \\
& \times\left(1-\left(\exp \left[-\left(c_{3} / y\right)^{b}\right]\right)^{\lambda}\right)^{c-1} ;
\end{aligned}
$$

$$
\begin{gathered}
f_{\text {OLLE-RW }}(z)=c_{2} c_{3} b a^{b} z^{-(b+1)} \exp \left[-c_{3}(a / y)^{b}\right] \\
\times\left(\exp \left[-c_{3}(a / y)^{b}\right]\left\{1-\exp \left[-c_{3}(a / y)^{b}\right]\right\}\right)^{-1+c_{2}} \\
\times\left(\exp \left[-c_{3} c_{2}(a / y)^{b}\right]+\left\{1-\exp \left[-c_{3}(a / y)^{b}\right]\right\}^{c_{2}}\right)^{-2}
\end{gathered}
$$

The parameters of the above densities are all positive real numbers except for the T-RW distribution for which $\left|c_{1}\right| \leq$ 1. The $1^{\text {st }}$ data set is an uncensored data set consisting of 100 observations on breaking stress of carbon fibers (in Gba) given by Nichols and Padgett (2006). Figure 5 gives the total time test (TTT) plot (See Aarset (1987)) for data set I. It indicates that the empirical H-R-Fs of data sets $\mathbf{I}$ is increasing. 
The $2^{\text {nd }}$ data set is generated data to simulate the strengths of glass fibers which was given by Smith and Naylor (1987). Figure 6 gives the TTT plot for data set II. It indicates that the empirical H-R-Fs of data sets II is increasing.

The $3^{\text {rd }}$ data set (wingo data) represents a complete sample from a clinical trial describe a relief time (in hours) for 50 arthritic patients. Many other usefull real data sets can be found and analyzed (see Basikhasteh et al. (2018), Elbiely and Yousof (2018 and 2019), Khalil et al. (2019), Gad et al. (2019), Ibrahim et al. (2019), Ibrahim (2019, 2020a, b), Ibrahim et al. (2020), Ibrahim and Yousof (2020), Mansour et al. (2020) and Al-Babtain et al. (2020a, b)). Figure 7 gives the TTT plot for data set III. It indicates that the empirical H-R-Fs of data sets III is increasing.

The statistics (C-V-M, A-D, K-S and P-value) of all fitted models are presented in Table 1, Table 3 and Table 5 for data set I, II and III respectively. The MLEs and corresponding standard errors (SEs) are given in Table 2, Table 4 and Table 6 for data set I, II and III respectively. Figure 8 gives the estimated density (E-P-D-F), Figure 9 gives the estimated C-D-F (E-C-D-F), Figure 10 gives the estimated H-R-F (E-H-R-F) and Figure 8 gives the P-P (P-P) plots. The GOLL-RE distribution in Tables 1, 3 and 5 give the lowest values the C-V-M, A-D, K-S and the biggest value of the P-value statistics as compared to other extensions of the RW models, and therefore the GOLL-RE can be chosen as the best model.

\section{Concluding remarks}

We introduce a new extension of the reciprocal Exponential distribution for modeling the extreme values. We used the Morgenstern family and the clayton copula for deriving many bivariate and multivariate extensions of the new model. Some of its mathematical properties are derived. We assessed the performance of the maximum likelihood estimators via a graphical simulation study. Based on the graphical simulations, the biases for each parameter are generally negative and decrease to zero as $n \rightarrow \infty$, the MSEs for each parameter decrease to zero as $n \rightarrow \infty$. The assessment was based on the sample size. The new reciprocal model is better than some other important competitive models in modeling the breaking stress data, the glass fibers data and the relief time data.

\section{References}

1. Aarset, M. V. (1987). How to identify a bathtub hazard rate. IEEE Transactions on Reliability, 36(1), 106-108.

2. Al-Babtain, A. A. Elbatal, I. and Yousof, H. M. (2020a). A new three parameter Fréchet model with mathematical properties and applications. Journal of Taibah University for Science, 14(1), 265-278.

3. Al-Babtain, A. A. Elbatal, I. and Yousof, H. M. (2020b). A new flexible three-parameter model: properties, Clayton Copula, and modeling real data, Symmetry, 12, 1-17. doi:10.3390/sym12030440

4. Basikhasteh, M., Lak, F., Alizadeh, M. and Yousof, H. M. (2018). The Odd Log-Logistic Generalized HalfNormal Lifetime Poisson Model, Pak. J. Stat. Oper. Res. 14(3), 111-128.

5. Brito, E., Cordeiro, G. M., Yousof, H. M., Alizadeh, M. and Silva, G. O. (2017). Topp-Leone Odd Log-Logistic Family of Distributions, Journal of Statistical Computation and Simulation, 87(15), 3040-3058.

6. Chakraborty, S., Handique, L., Altun, E. and Yousof, H. M. (2018). A new statistical model for extreme values: mathematical properties and applications. International Journal of Open Problems in Computer Science and Mathematics, 12(1), 1-18.

7. Cordeiro, G. M., Alizadeh, M. M., Ozel, G., Hosseini, B., Ortega, E. M. M. and Altun, E. (2016). The generalized odd log-logistic family of distributions: properties, regression models and applications, Journal of Statistical Computation and Simulation, 87(5), 908-932.

8. Cordeiro, G. M., Yousof, H. M., Ramires, T. G. and Ortega, E. M. M. (2018). The Burr XII system of densities: properties, regression model and applications. Journal of Statistical Computation and Simulation, 88(3), 432456.

9. Elbiely, M. M. and Yousof, H. M. (2018). A new extension of the Lomax distribution and its applications, Journal of Statistics and Applications, 2(1), 18-34.

10. Elbiely, M. M. and Yousof, H. M. (2019). A new inverse Weibull distribution: properties and applications. Journal of Mathematics and Statistics, 15(1), 30-43.

11. Fréchet, M. (1927). Sur la loi de probabilité de lécart maximum. Ann. de la Soc. polonaisede Math, 6, 93--116.

12. Gleaton, J. U. and Lynch, J. D. (2006). Properties of generalized loglogistic families of lifetime distributions. Journal of Probability and Statistical Science, 4, 51-64.

13. Gad, A. M., Hamedani, G. G., Salehabadi, S. M. and Yousof, H. M. (2019). The Burr XII-Burr XII distribution: mathematical properties and characterizations. Pakistan Journal of Statistics, 35(3), 229-248.

14. Goual, H. and Yousof, H. M. (2019). Validation of Burr XII inverse Rayleigh model via a modified chi-squared goodness-of-fit test. Journal of Applied Statistics, 47(1), 1-32. 
15. Gupta, R. C. and Gupta, R. D. (2007). Proportional reversed hazard rate model and its applications. J. Statist Plan Inference.137, 3525--3536.

16. Hamedani G. G., Altun, E, Korkmaz, M. C., Yousof, H. M. and Butt, N. S. (2018). A new extended G family of continuous distributions with mathematical properties, characterizations and regression modeling. Pak. J. Stat. Oper. Res., 14 (3), 737-758.

17. Hamedani G. G. Rasekhi, M., Najibi, S. M., Yousof, H. M. and Alizadeh, M., (2019). Type II general exponential class of distributions. Pak. J. Stat. Oper. Res., XV(2), 503-523.

18. Hamedani G. G. Yousof, H. M., Rasekhi, M., Alizadeh, M. and Najibi, S. M. (2017). Type I general exponential class of distributions. Pak. J. Stat. Oper. Res., XIV(1), 39-55.

19. Ibrahim, M. (2019). A new extended Fréchet distribution: properties and estimation. Pak. J. Stat. Oper. Res., 15 (3), 773-796.

20. Ibrahim, M. (2020a). The compound Poisson Rayleigh Burr XII distribution: properties and applications. Journal of Applied Probability and Statistics, 15(1), 73-97.

21. Ibrahim, M. (2020b). The generalized odd Log-logistic Nadarajah Haghighi distribution: statistical properties and different methods of estimation. Journal of Applied Probability and Statistics, forthcoming.

22. Ibrahim, M., Altun, E. and Yousof, H. M. (2020). A new distribution for modeling lifetime data with different methods of estimation and censored regression modeling. Statistics, Optimization and Information Computing, $8,610-630$.

23. Ibrahim, M. and Yousof, H. M. (2020). A new generalized Lomax model: statistical properties and applications, Journal of Data Science, 18(1), $190-217$.

24. Ibrahim, M., Yadav, A. S. Yousof, H. M., Goual, H. and Hamedani, G. G. (2019). A new extension of Lindley distribution: modified validation test, characterizations and different methods of estimation, Communications for Statistical Applications and Methods, 26(5), 473-495.

25. Khalil, M. G., Hamedani G. G. and Yousof, H. M. (2019). The Burr X exponentiated Weibull model: characterizations, mathematical properties and applications to failure and survival times data. Pak. J. Stat. Oper. Res., XV(1), 141-160.

26. Korkmaz, M. C., Alizadeh, M., Yousof, H. M. and Butt, N. S. (2018). The generalized odd Weibull generated family of distributions: statistical properties and applications. Pak. J. Stat. Oper. Res., 14 (3), 541-556.

27. Korkmaz, M. C., Altun, E., Yousof, H. M. and Hamedani G. G. (2019). The Odd Power Lindley Generator of Probability Distributions: Properties, Characterizations and Regression Modeling, International Journal of Statistics and Probability, 8(2). 70-89.

28. Korkmaz, M. C. Yousof, H. M. and Ali, M. M. (2017). Some Theoretical and Computational Aspects of the Odd Lindley Fréchet Distribution, Journal of Statisticians: Statistics and Actuarial Sciences, 2, 129-140.

29. Mansour, M., Yousof, H. M., Shehata, W. A. M. and Ibrahim, M. (2020). A new two parameter Burr XII distribution: properties, copula, different estimation methods and modeling acute bone cancer data, Journal of Nonlinear Science and Applications, 13, 223-238.

30. Nichols, M. D, Padgett, W. J. (2006). A Bootstrap control chart for Weibull percentiles. Quality and Reliability Engineering International, 22, 141-151.

31. Smith, R. L. and Naylor, J. C. (1987). A comparison of maximum likelihood and bayesian estimators for the three-parameter Weibull distribution. Applied Statistics, 36, 358-369.

32. Yousof, H. M., Afify, A. Z., Alizadeh, M., Butt, N. S., Hamedani, G. G. and Ali, M. M. (2015). The transmuted exponentiated generalized-G family of distributions. Pak. J. Stat. Oper. Res., 11, 441-464.

33. Yousof, H. M., Afify, A. Z., Ebraheim, A. N., Hamedani, G. G. and Butt, N. S. (2016). On six-parameter Fréchet distribution: properties and applications, Pak. J. Stat. Oper. Res., 12, 281-299.

34. Yousof, H. M., Alizadeh, M., Jahanshahi and, S. M. A., Ramires, T. G., Ghosh, I. and Hamedani G. G. (2017). The transmuted Topp-Leone $\mathrm{G}$ family of distributions: theory, characterizations and applications, Journal of Data Science. 15, 723-740.

35. Yousof, H. M., Altun, E. and Hamedani, G. G. (2018 a). A new extension of Frechet distribution with regression models, residual analysis and characterizations. Journal of Data Science, 16(4), 743-770.

36. Yousof, H. M., Jahanshahi, S. M., Ramires, T. G Aryal, G. R. and Hamedani G. G. (2018 b). A new distribution for extreme values: regression model, characterizations and applications. Journal of Data Science, 16(4), 677706. 
Appendix: A

Table 1: C-V-M, A-D, K-S and P-value for data set I.

\begin{tabular}{ccccc}
\hline Model & \multicolumn{4}{c}{ Goodness of fit criteria } \\
\hline GOLL-RE & C-V-M & A-D & K-S & P-value \\
\hline OLLE-RW & 0.069 & 0.577 & 0.0555 & 0.9177 \\
OLLE-RR & 0.1553 & 0.9639 & 0.5561 & $2.2 \times \mathrm{e}^{-16}$ \\
OLL-RR & 0.15532 & 1.21197 & 0.65497 & $2.2 \times \mathrm{e}^{-16}$ \\
RW & 0.109 & 0.7657 & 0.6550 & $2.2 \times \mathrm{e}^{-16}$ \\
K-RW & 0.0812 & 0.6217 & 0.0759 & 0.4282 \\
E-RW & 0.1091 & 0.7658 & 0.0874 & 0.6118 \\
BRW & 0.0809 & 0.6207 & 0.0757 & 0.6147 \\
T-RW & 0.0871 & 0.6209 & 0.0782 & 0.5734 \\
MO-RW & 0.0886 & 0.6142 & 0.0763 & 0.5168 \\
Mc-RW & 0.1333 & 1.0608 & 0.0807 & 0.5332 \\
\hline
\end{tabular}

Table 2: MLEs and their SEs for data set I.

\begin{tabular}{|c|c|c|c|c|c|}
\hline \multirow[t]{2}{*}{ Model } & \multicolumn{5}{|c|}{ Estimates } \\
\hline & $\mathrm{c}_{1}$ & $\mathrm{c}_{2}$ & $\mathrm{c}$ & $c_{3}$ & $\mathrm{~b}$ \\
\hline \multirow{2}{*}{ GOLL-RE } & 4.979 & 2.3994 & & 0.4472 & \\
\hline & $(0.4195)$ & $(0.000)$ & & $(0.000)$ & \\
\hline \multirow[t]{2}{*}{ OLLE-RW } & 0.1351 & & 3.7216 & 0.9296 & 21.319 \\
\hline & $(0.011)$ & & $(0.0034)$ & $(0.0033)$ & $(0.0034)$ \\
\hline \multirow[t]{2}{*}{ OLLE-RR } & 0.4946 & & 0.067 & 1.74262 & \\
\hline & $(0.0414)$ & & $(0.7195)$ & (9.3007) & \\
\hline \multirow{2}{*}{ OLL-RR } & 0.49459 & & & 0.45242 & \\
\hline & 0.04135 & & & 0.03869 & \\
\hline \multirow[t]{2}{*}{ RW } & & & & 1.3968 & 4.3724 \\
\hline & & & & $(0.0336)$ & $(0.3278)$ \\
\hline \multirow[t]{2}{*}{ K-RW } & & 0.8489 & 1.6239 & 1.6341 & 3.4208 \\
\hline & & (16.083) & $(0.6979)$ & (9.049) & $(0.7635)$ \\
\hline \multirow[t]{2}{*}{ E-RW } & & 0.9395 & & 1.4169 & 0.9395 \\
\hline & & (3.543) & & $(2.568)$ & $(0.3278)$ \\
\hline \multirow[t]{2}{*}{ B-RW } & & 0.7346 & 1.5830 & 1.6684 & 3.5112 \\
\hline & & $(1.5290)$ & $(0.7132)$ & $(0.7662)$ & $(0.9683)$ \\
\hline \multirow[t]{2}{*}{ T-RW } & -0.7166 & & & 1.2656 & 4.7121 \\
\hline & $(0.2616)$ & & & $(0.0579)$ & $(0.3657)$ \\
\hline \multirow[t]{2}{*}{ MO-RW } & & 0.0033 & & 6.2296 & 1.2419 \\
\hline & & $(0.0009)$ & & (1.0134) & $(0.1181)$ \\
\hline \multirow[t]{2}{*}{ Mc-RW } & 0.8503 & 44.423 & 19.859 & 0.0203 & 46.974 \\
\hline & $(0.1353)$ & $(25.100)$ & $(6.706)$ & $(0.0060)$ & (21.871) \\
\hline
\end{tabular}

Table 3: C-V-M, A-D, K-S and P-value for data set II.

\begin{tabular}{ccccc}
\hline Model & \multicolumn{4}{c}{ Goodness of fit criteria } \\
\hline GOLL-RE & C-V-M & A-D & K-S & P-value \\
OLLE-RW & 0.0626 & 0.539 & 0.0677 & 0.9158 \\
OLLE-RR & 0.10487 & 0.8325 & 0.55196 & $6.66 \times \mathrm{e}^{-16}$ \\
OLL-RR & 0.1502 & 1.14697 & 0.67949 & $6.66 \times \mathrm{e}^{-16}$ \\
RW & 0.15021 & 1.14697 & 0.67951 & $6.66 \times \mathrm{e}^{-16}$ \\
K-RW & 0.0707 & 0.5332 & 0.0772 & 0.8185 \\
E-RW & 0.0634 & 0.4981 & 0.0715 & 0.8810 \\
B-RW & 0.0707 & 0.5332 & 0.0772 & 0.8187 \\
T-RW & 0.0640 & 0.5008 & 0.0716 & 0.8804 \\
MO-RW & 0.0655 & 0.4939 & 0.0735 & 0.8470 \\
Mc-RW & 0.0629 & 0.4902 & 0.0813 & 0.7685 \\
\end{tabular}


Table 4: MLEs and their SEs for data set II.

\begin{tabular}{|c|c|c|c|c|c|}
\hline \multirow[t]{2}{*}{ Model } & \multicolumn{5}{|c|}{ Estimates } \\
\hline & $\mathrm{c}_{1}$ & $\mathrm{c}_{2}$ & $\mathrm{c}$ & $\mathrm{c}_{3}$ & $\mathrm{~b}$ \\
\hline \multirow[t]{2}{*}{ GOLL-RE } & 6.0667 & 2.9137 & & 0.364 & \\
\hline & $(0.6449)$ & $(0.000)$ & & $(0.000)$ & \\
\hline \multirow[t]{2}{*}{ OLLE-RW } & 0.1449 & & 0.00879 & 1.2997 & 24.878 \\
\hline & $(0.0129)$ & & $(0.000)$ & (0.000) & $(0.000)$ \\
\hline \multirow[t]{2}{*}{ OLLE-RR } & 0.5025 & & 0.0716 & 1.7048 & \\
\hline & $(0.0529)$ & & (1.13062) & (13.47) & \\
\hline \multirow[t]{2}{*}{ OLL-RR } & 0.50251 & & & 0.45599 & \\
\hline & 0.052946 & & & 0.048652 & \\
\hline \multirow[t]{2}{*}{ RW } & & & & 1.4108 & 5.4377 \\
\hline & & & & $(0.0344)$ & $(0.5192)$ \\
\hline \multirow{2}{*}{ K-RW } & & 0.2855 & 1.2824 & 1.9142 & 4.7731 \\
\hline & & (9.1338) & $(0.6388)$ & (12.836) & (1.3134) \\
\hline \multirow[t]{2}{*}{ E-RW } & & 0.9059 & & 1.4367 & 5.4379 \\
\hline & & (2.764) & & $(4.324)$ & $(0.5193)$ \\
\hline \multirow[t]{2}{*}{ B-RW } & & 1.2996 & 1.2649 & 1.3945 & 4.7927 \\
\hline & & (4.4378) & $(0.6640)$ & $(0.9304)$ & (1.4641) \\
\hline \multirow[t]{2}{*}{ T-RW } & 0.7778 & & & 1.5491 & 4.3139 \\
\hline & $(0.2477)$ & & & $(0.0655)$ & $(0.5849)$ \\
\hline \multirow[t]{2}{*}{ MO-RW } & & 0.0023 & & 5.2383 & 1.4537 \\
\hline & & $(0.0004)$ & & $(0.8209)$ & $(0.1650)$ \\
\hline \multirow[t]{2}{*}{ Mc-RW } & & 56.227 & 14.953 & 0.0073 & 29.104 \\
\hline & & $(30.539)$ & $(4.733)$ & $(0.0013)$ & (11.304) \\
\hline
\end{tabular}

Table 5: C-V-M, A-D, K-S and P-value for data set III.

\begin{tabular}{ccccc}
\hline Model & \multicolumn{4}{c}{ Goodness of fit criteria } \\
\hline GOLL-RE & C-V-M & A-D & K-S & P-value \\
RW & 0.13558 & 1.00852 & 0.1082 & 0.6016 \\
E-RW & 0.3233 & 2.0301 & 0.1506 & 0.2066 \\
T-RW & 0.3233 & 2.0301 & 0.1506 & 0.2064 \\
\hline
\end{tabular}

Table 6: MLEs and their SEs for data set III.

\begin{tabular}{cccccc}
\hline Model & \multicolumn{5}{c}{ Estimates } \\
\hline GOLL-RE & $\mathrm{c}_{1}$ & $\mathrm{c}_{2}$ & $\mathrm{c}$ & $\mathrm{c}_{3}$ & $\mathrm{~b}$ \\
& 4.1213 & 0.12759 & & 3.1233 & \\
RW & $(0.4856)$ & $(0.4195)$ & & $(10.269)$ & 3.2078 \\
& & & 0.4859 & $(0.0227)$ & $(0.3263)$ \\
E-RW & & & 0.5013 & 3.2077 \\
T-RW & & & 0.9047 & $(3.244)$ & $(0.3263)$ \\
E-RW & -0.5816 & $(18.784)$ & 0.4400 & 3.4974 \\
\end{tabular}




\section{Appendix B}
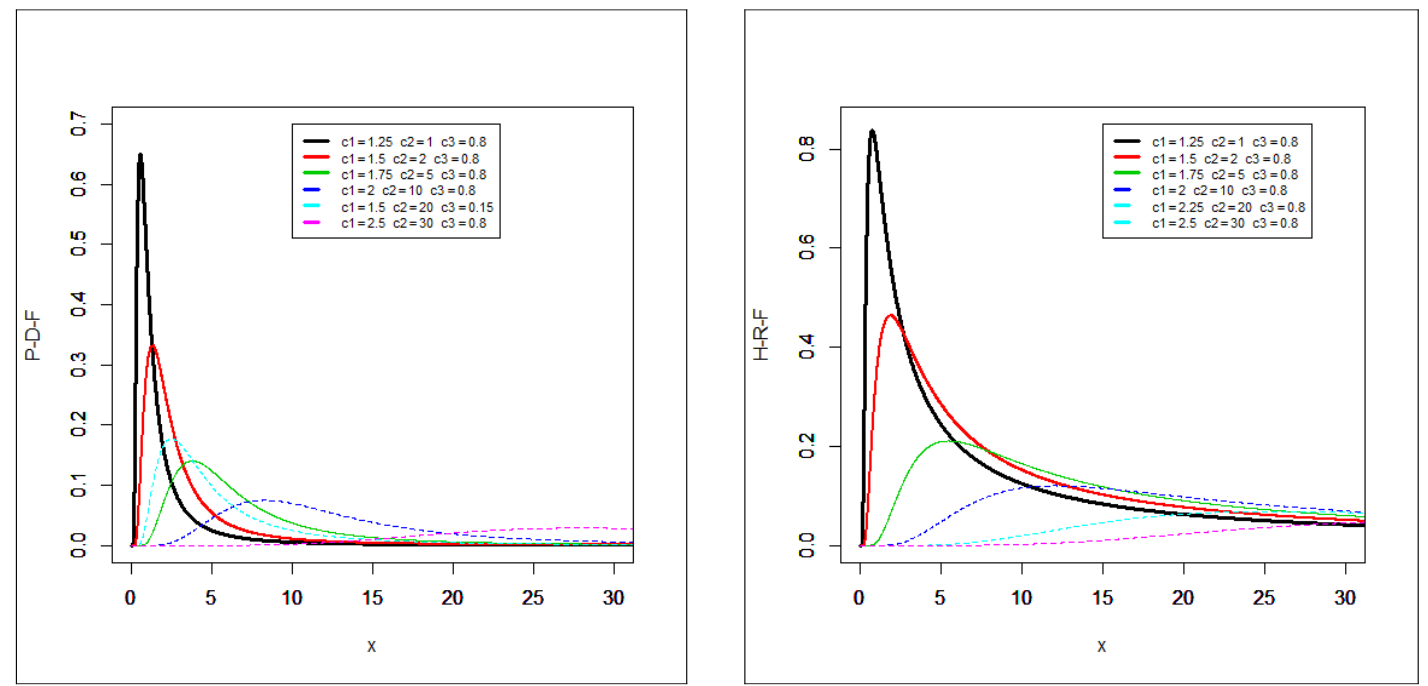

Figure 1: Plots of the GOLL-RE P-D-F and H-R-F.

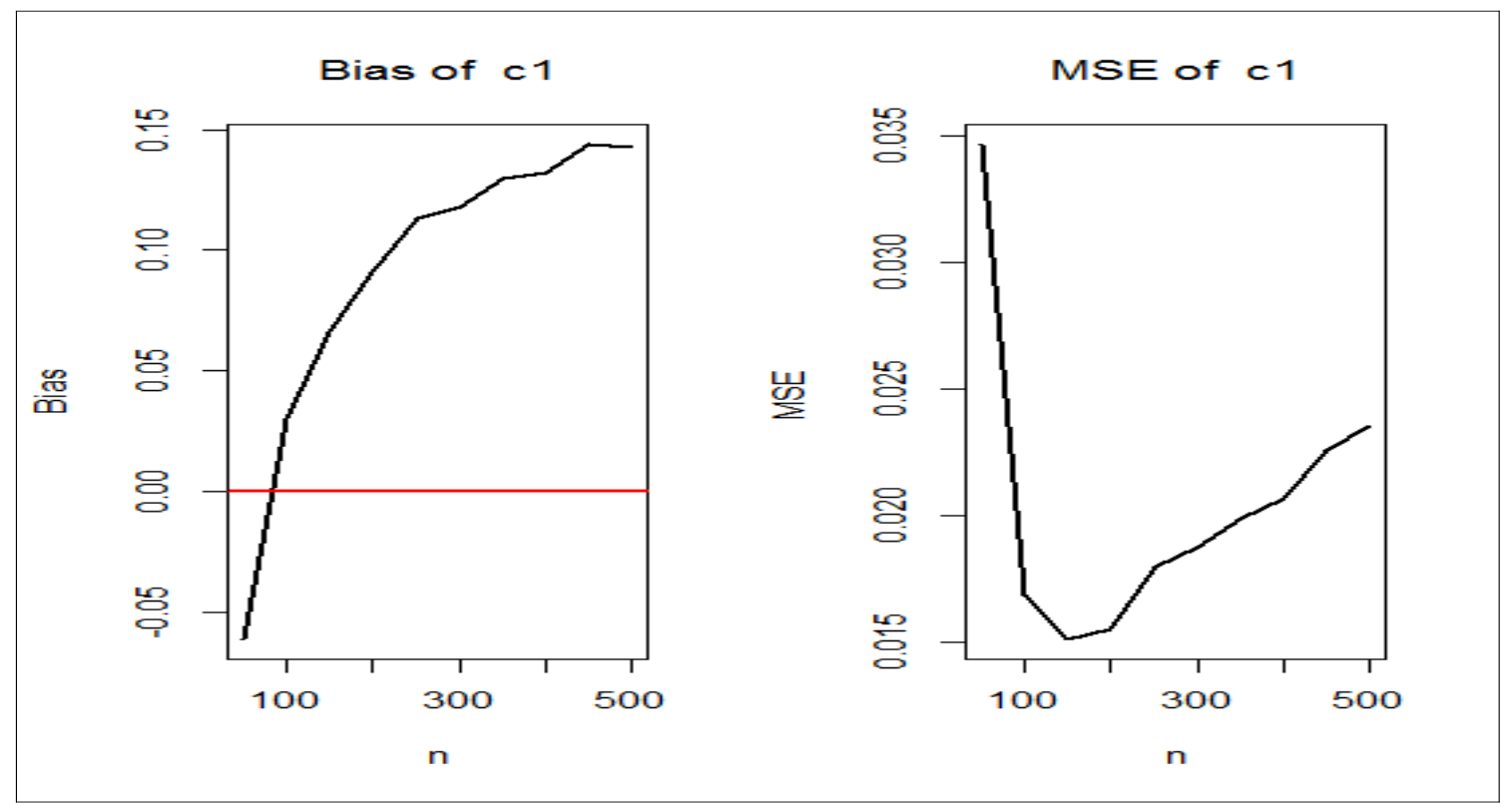

Figure 2: Biases and MSEs for $\mathrm{c}_{1}$ and $\mathrm{n}=50,100, \ldots, 500$ for the GOLL-RE model. 


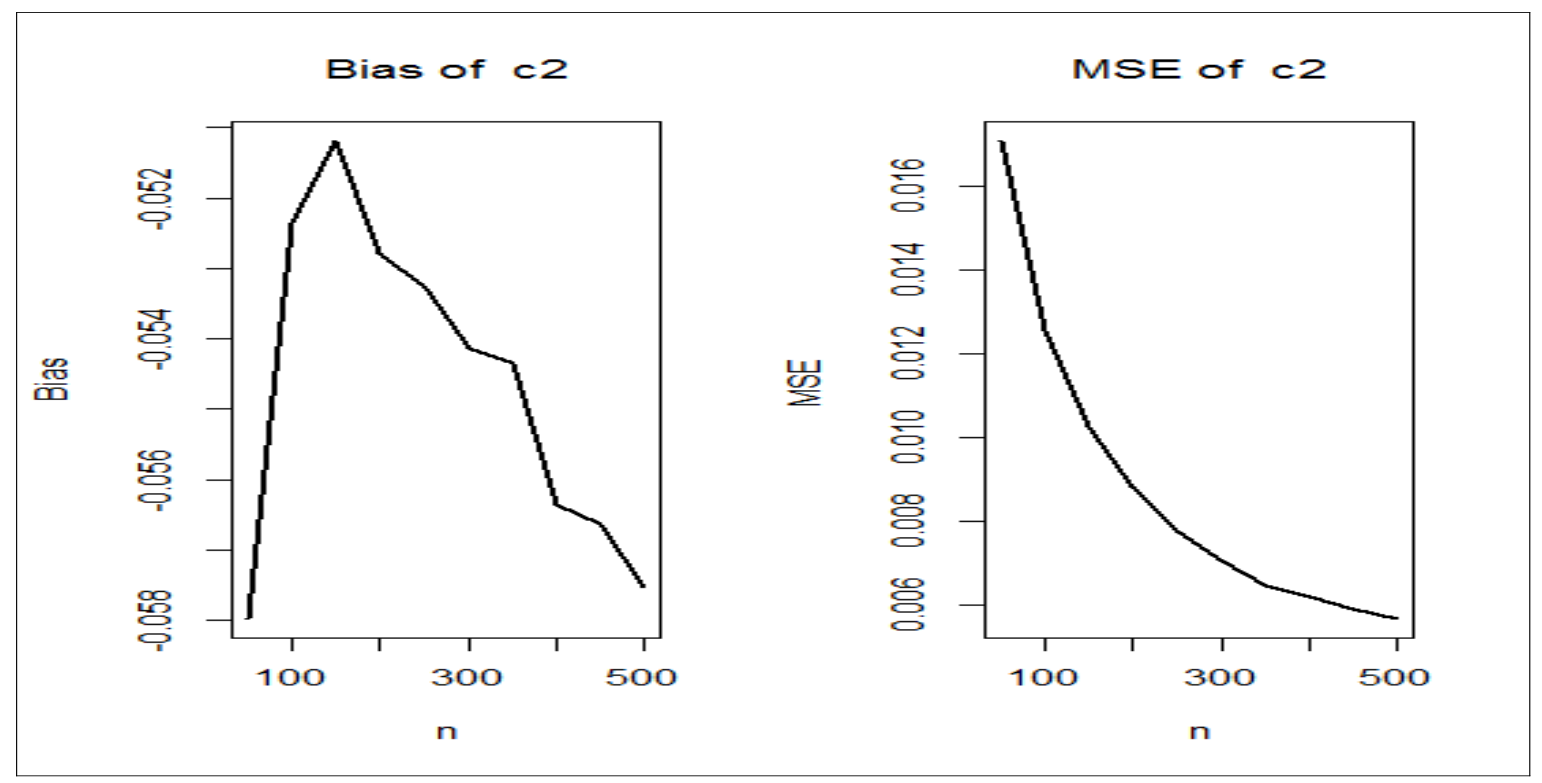

Figure 3: Biases and MSEs for $c_{2}$ and $n=50,100, \ldots, 500$ for the GOLL-RE model.

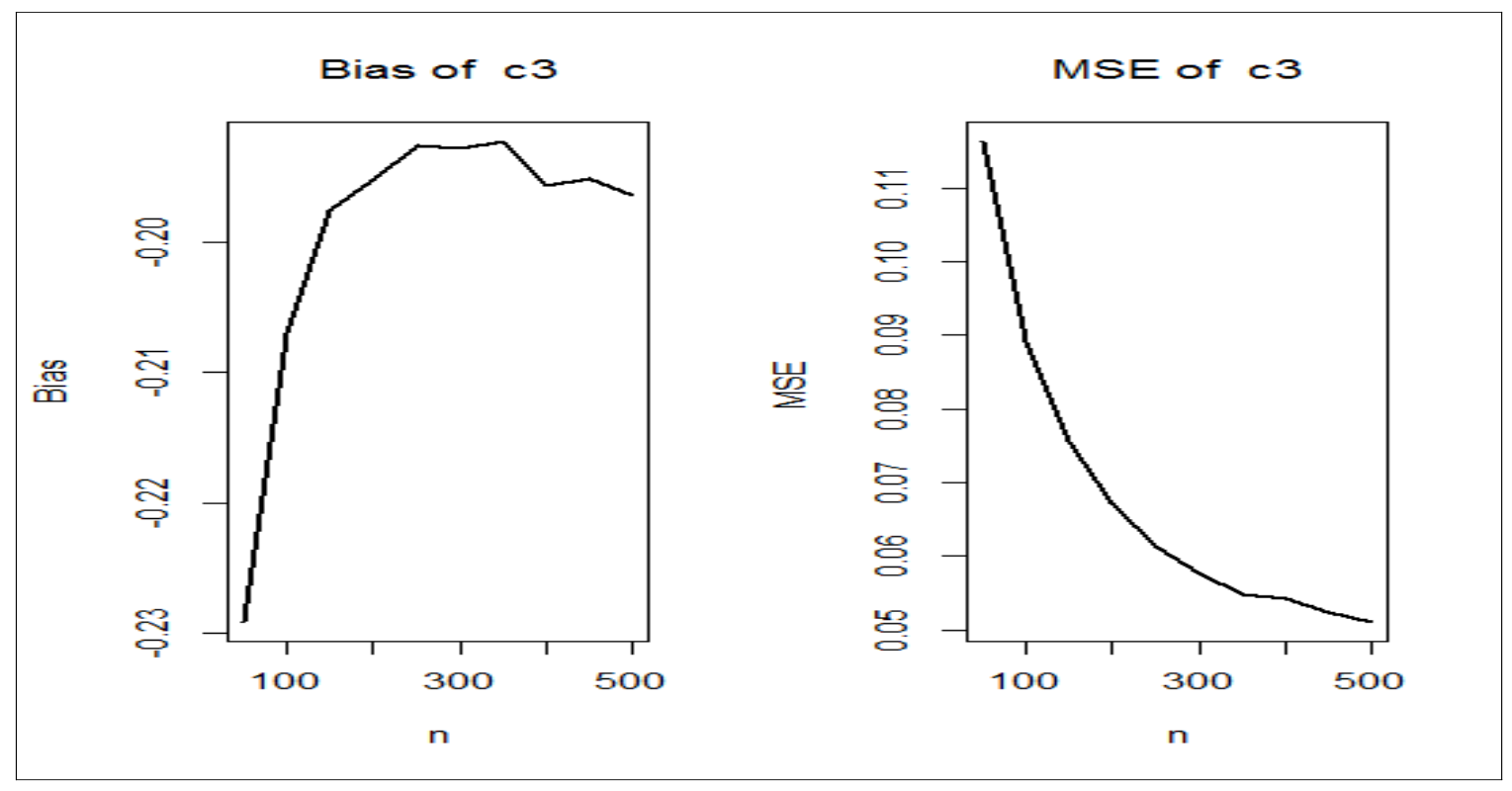

Figure 4: Biases and MSEs for $c_{3}$ and $n=50,100, \ldots, 500$ for the GOLL-RE model. 


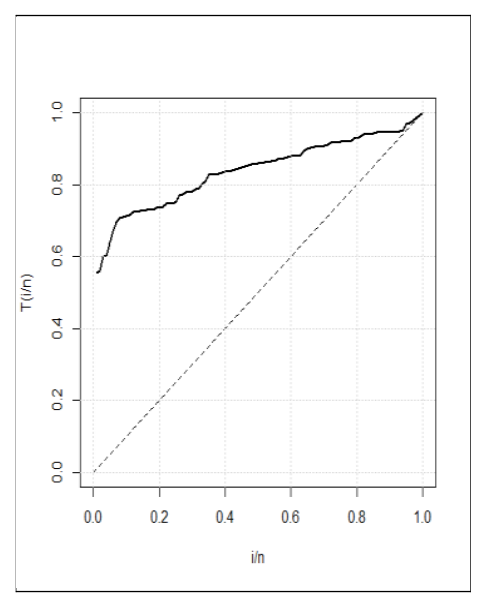

Figure 5: TTT plot for data set $\mathbf{I}$.
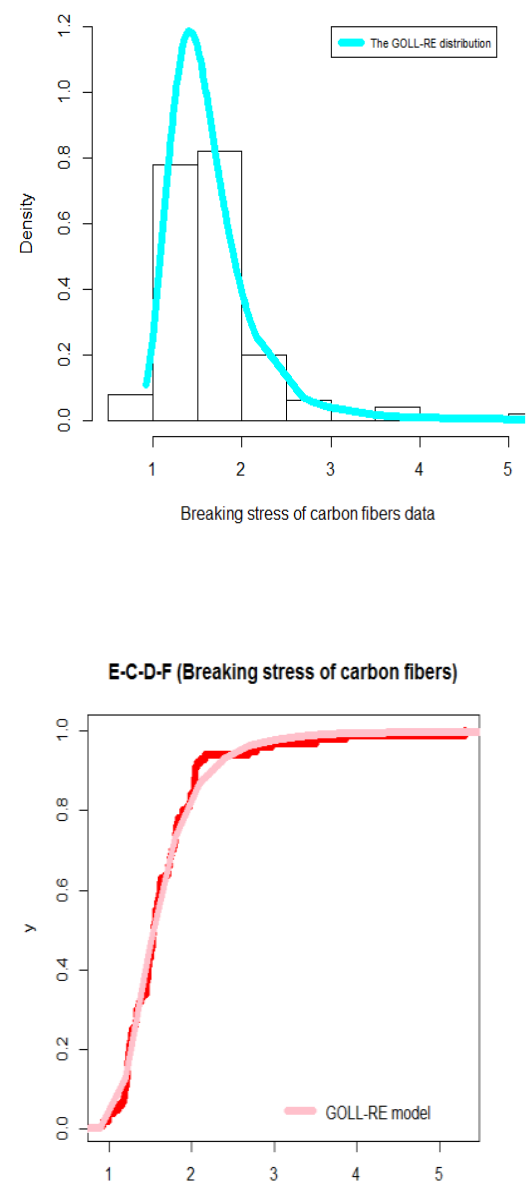

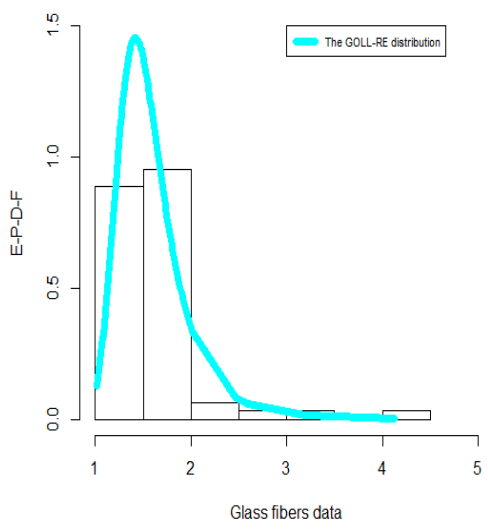

Figure 8: E-P-D-Fs.

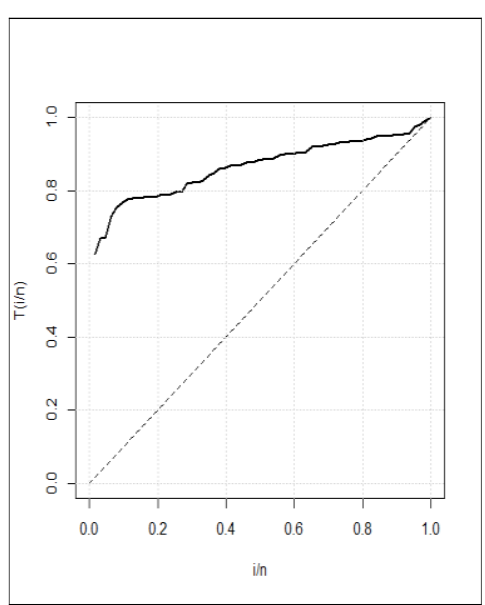

Figure 6: TTT plot for data set II.

Figure 7: TTT plot for data set III.
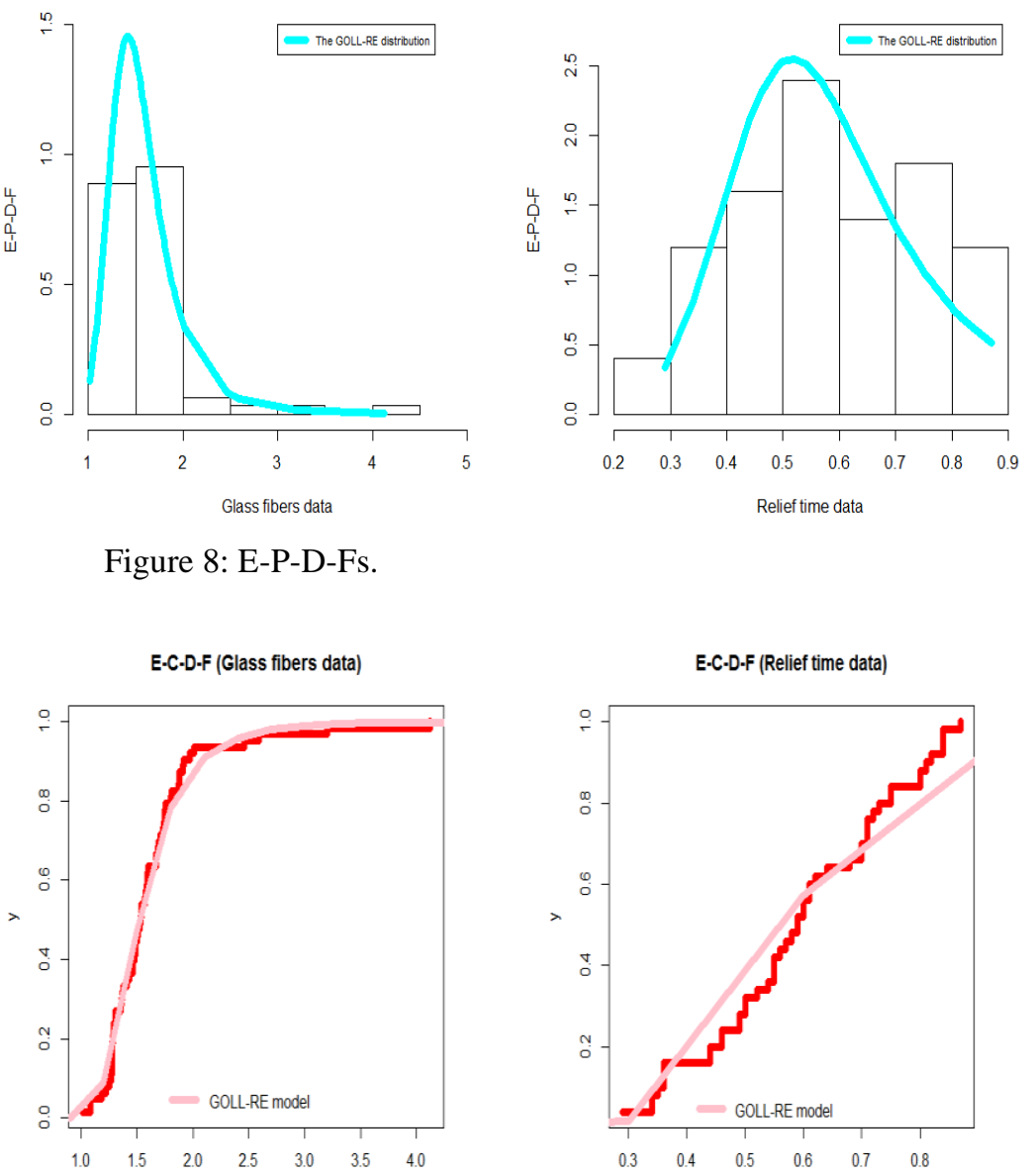

Figure 9: E-C-D-Fs. 

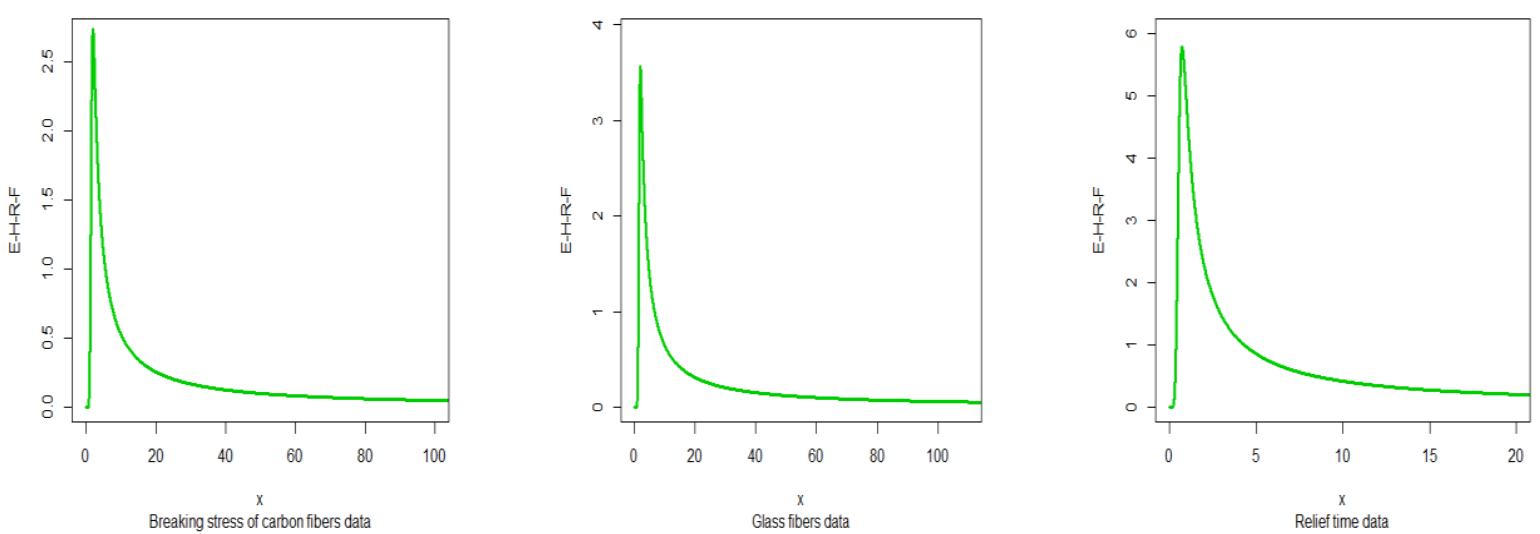

Figure 10: E-H-R-Fs.
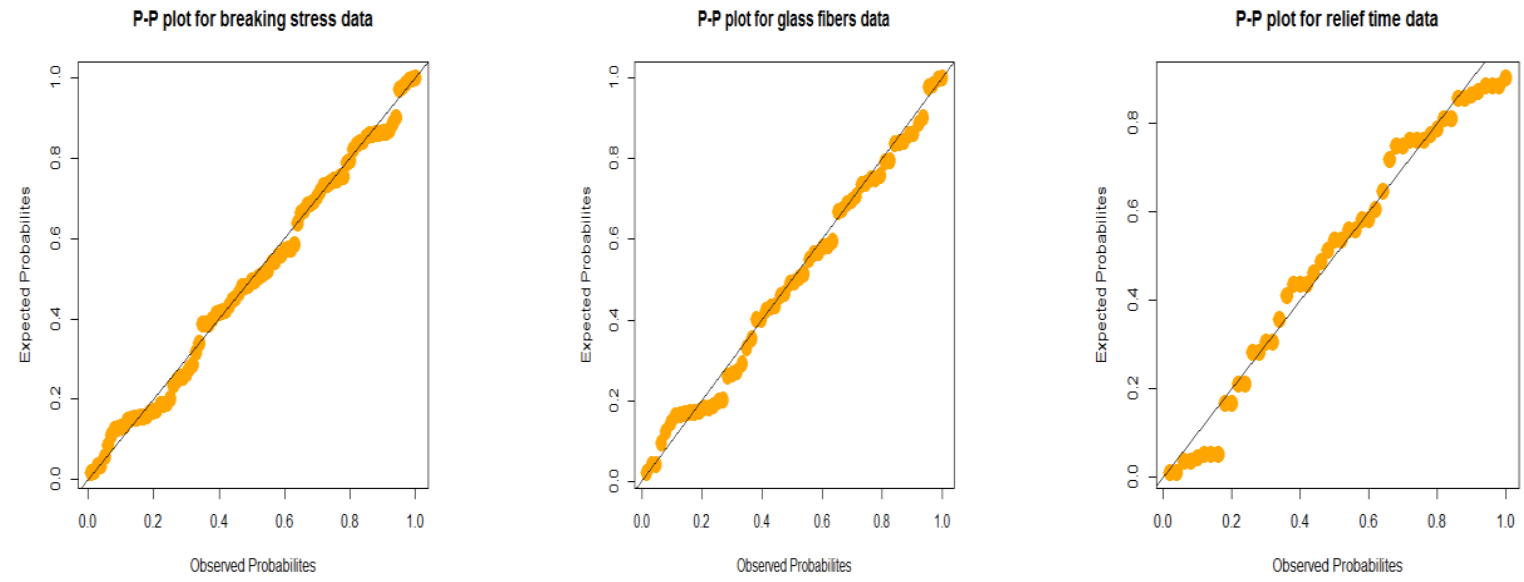

Figure 11: P-P plots. 